\title{
Germanica
}

\section{Ein Intellektueller als Grenzgänger. Zu Hugo Loetschers «Immunen»}

\section{Rosmarie Zeller}

\section{Q OpenEdition}

1 Journals

Édition électronique

URL : http://journals.openedition.org/germanica/2494

DOI : 10.4000/germanica.2494

ISSN : 2107-0784

Éditeur

Université de Lille

\section{Édition imprimée}

Date de publication : 30 juin 1990

Pagination : 67-76

ISSN : 0984-2632

\section{Référence électronique}

Rosmarie Zeller, «Ein Intellektueller als Grenzgänger. Zu Hugo Loetschers «Immunen» », Germanica [Online], 7 | 1990, Online erschienen am: 17 Juli 2014, abgerufen am 06 Oktober 2020. URL : http:// journals.openedition.org/germanica/2494; DOI : https://doi.org/10.4000/germanica.2494

Ce document a été généré automatiquement le 6 octobre 2020.

(c) Tous droits réservés 


\title{
Ein Intellektueller als Grenzgänger. $\mathrm{Zu}$ Hugo Loetschers «Immunen»
}

\author{
Rosmarie Zeller
}

1 Ich möchte meine Ausführungen zu Hugo Loetschers Figur des Immunen als eines Grenzgängers in den Rahmen einiger allgemeiner Überlegungen zum Problem der Grenze in der modernen Literatur stellen. Soweit ich sehe, hat vor allem der russische Semiotiker Jurij M. Lotman über die Rolle der Grenze im literarischen Text nachgedacht ${ }^{1}$. Im Normalfall wird die dargestellte Welt in zwei Teile aufgeteilt, welche durch eine Grenze getrennt sind. Es gibt Figuren, die an den einen der beiden Teile gebunden sind, während der Held als dynamische Figur die Grenze überschreiten kann. Das Ereignishafte des Textes oder das Sujet, wie Lotman sagt, ist die Entfaltung dieser Grenzüberschreitung, welche nicht in einem einmaligen Akt geschehen muss, sondern auch in einer Hierarchie von Sujetbewegungen geschehen kann. Das klassische Schema dieser Grenzüberschreitung besteht darin, dass der Held von einer Welt A durch Überschreiten einer Grenze in eine Welt B gelangt, wo er einige Abenteuer zu bestehen hat, um dann am Ende wieder in die Welt A zurückzukehren.

2 Als Illustration dieser semantischen Organisation kann der noch ganz den Gestaltungsprinzipien des realistischen Romans verpflichtete Zauberberg gelten, wo die beiden semantischen Welten, wie übrigens immer bei Thomas Mann, in einem topologischen Gegensatz, in diesem Fall im Gegensatz des Flachlandes und der Bergwelt abgebildet werden. Die Bergwelt, welche mit den Attributen der Unterwelt und des Todes versehen ist, wird, wie wir dies aus der Mythologie kennen, durch ein Wasser, in diesem Fall durch den Bodensee, von der Welt des Flachlandes abgetrennt. Hans Castorp ist der Held, welcher diese Grenze überschreitet und hier stufenweise in die Eigenheiten der Bergwelt eindringt, um am Ende seines Lebens wieder in die bürgerliche Welt des Flachlands zurückzukehren. Man hat deshalb nicht zu Unrecht den Zauberberg einen Bildungsroman genannt.

3 Eine solche Grenze setzt voraus, dass es im Text mindestens zwei einander entgegengesetzte semantische Welten gibt. Im modernen Roman, der nicht mehr der realistischen Darstellungsweise verpflichtet ist und der auf die Vermittlung von Werten 
verzichtet, kann es Grenzen in diesem Sinn nicht mehr geben. An die Stelle einer durch Grenzen unterteilten Welt tritt häufig ein anderes topologisches Modell: das Labyrinth. Man kann als Beispiele RobbeGrillets Roman Dans le labyrinthe nennen, Pingets Inquisitoire, wo ein Haus zum Labyrinth wird oder Kafkas Raumgestaltung z.B. in der Erzählung Der Bau oder im Schloss. Meine These ist, dass es im modernen Roman weniger darum geht, Grenzen zu setzen als Grenzen aufzulösen, die Erfahrung darzustellen, dass es keine festen Grenzen mehr gibt, dass Grenzen eine optische Illusion sind. Döblin hat im Zusammenhang mit Berlin Alexanderplatz gesagt, er habe bei seiner Arbeit als Arzt erkannt, «wie es da keine so straffe formulierbare Grenze zwischen Kriminellen und NichtKriminellen gibt $»^{2}$. Musils auf moralischem Gebiet in dieselbe Richtung gehenden Überlegungen sind bekannt. Schon in den Verwirrungen des Zöglings Törless hat er die Erfahrung der verwischten Grenzen dargestellt: Die Verwirrungen von Törless bestehen ja gerade darin, dass dieGrenze zwischen einer Welt, die der bürgerlichen Moral und der Rationalität entspricht, und einer Welt der moralischen Übertretungen und der Irrationalität aufgelöst wird. So heisst es am Ende von Törless:

Er wusste nun zwischen Tag und Nacht zu scheiden; - er hatte es eigentlich immer gewusst, und nur ein schwerer Traum war verwischend über diese Grenzen hingeflutet, und er schämte sich dieser Verwirrung: aber die Erinnerung, dass es anders sein kann, dass es feine leicht verlöschbare Grenzen rings um den Menschen gibt, [...] auch, diese Erinnerung hatte sich tief in ihn gesenkt undstrahlte blasse Schatten aus ${ }^{3}$.

4 Dieser Erfahrung der sich verwischenden Grenzen entspricht auf der topographischen Ebene, dass die Dachkammer, in der die sexuellen Verfehlungen stattfinden, sich inmitten des Internats befindet. Das wesentliche Leben, welches Ulrich anstrebt, ist dadurch gekennzeichnet, dass «alles grenzenlos in dich übergeht», wie Ulrich, der Mann ohne Eigenschaften einmal sagt ${ }^{4}$. Türen und Pässe, welche ihrer Natur nach Durchund Übergänge sind, spielen deshalb in Musils Werk eine wichtige Rolle. Ganz ähnlich gibt es in Kafkas Werk keine Grenzen mehr zwischen verschiedenen Bereichen, man denke nur an den Prozess, wo das Gericht überall ist, so dass es keine bestimmten Orte für bestimmte Funktionen mehr gibt.

5 Proust stellt in À la recherche du temps perdu die Grenze nals optische Illusion des Helden dar. Erst gegen Ende des Romans erkennt der Held, dass der schönste Spaziergang nach Guermantes über Méséglise führt, dass also zwischen den zwei «Seiten» nicht jene unüberwindliche Grenze besteht, wie er sie bis dahin angenommen hatte ${ }^{5}$. Ebenso lernt er im Laufe des Romans die Durchlässigkeit der Grenzen zwischen gesellschaftlichen Gruppen kennen. In Handkes Werk ist der Übergang ein wichtiges Motiv sowohl in bezug auf die künstlerische Gestaltung wie in bezug auf das Verhältnis der Figuren zur Welt. So ist z.B. die Schwelle ein zentrales Motiv in Der Chinese des Schmerzes.

$6 \mathrm{Zu}$ diesen Autoren, welche sich nicht mit der Setzung von Grenzen, sondern mit deren Auflösung befassen, gehört auch Hugo Loetscher, der nicht zu Unrecht als der kosmopolitischste unter den Schweizer Autoren gilt. Loetscher ist ein grosser Reisender und schon von daher ein Grenzüberschreiter. In seinem Werk hat er immer wieder auch aussereuropäische Länder dargestellt, so in Wunderwelt den Nordosten Brasiliens, in Herbst in der grossen Orange Los Angeles. Der Roman Die Papiere des Immunen kann unter anderem als Weltreise gelesen werden, die von der engeren Heimat des Entlebuch nach Paris, London, Portugal, Brasilien und Thailand führt. Loetscher gestaltet in seinen literarischen Texten eine wichtige Erfahrung des modernen Men 
sehen, nämlich dass ihm einerseits durch die Medien und andererseits durch die modernen Transportmittel die ganze Welt offensteht: dass das Fremde zum täglichen Leben des modernen Menschen gehört. Wenn eine Frau das Frühstück für einen Mann zubereitet, bietet sie dazu, wie Loetscher uns darlegt, die ganze Welt auf.

Die Geschichte dieses Frühstücks hätte auf einer amerikanischen Maisfarm oder in einer spanischen Orangenplantage beginnen können; auf einem Getreidefeld oder einem Zuckerrübenacker. [...] Für die Filtertüte war Holz gefällt worden [...] Bauxit hatte abgebaut werden müssen, damit die Frau vom Yoghurtbecher einen Deckel abreissen konnte. Damit dieser Tisch so gedeckt werden konnte, wie die Frau es sich wünschte, waren Container gestapelt und Kisten verladen worden, Kräne und Roboter waren zum Einsatz gekommen [...]. Im skandinavischen Norden waren Wälder abgeholzt und im Nahen Osten öltanker vollgepumpt und in Übersee Plantagen angelegt worden, durch halb Europa eine Leitung geführt und in den Bergen ein See gestaut worden... / Eine ganze Welt war nötig6.

7 Loetschers Mensch kann nicht einmal ein Frühstück zubereiten, ohne die ganze Welt aufzubieten, die immer wieder als eine Welt ohne Grenzen dargestellt wird.

Es mag auf den ersten Blick wenig sinnvoll scheinen im rahmen eines Kolloquiums über «Grenze und Entgrenzung» einen Autor zu wählen, für den es offenbar keine Grenzen gibt. Die Grenzenlosigkeit der Welt ist für Loetscher nicht selbstverständlich, die Auflösung der Grenzen, die Erfahrung der Entgrenzung, um mit dem Litel des Kolloquiums zu sprechen, wird ständig thematisiert.

So sind z.B. nicht einmal Loetschers Werke begrenzt, aus dem Immunen sind die Papiere des Immunen und die Tiergeschichten Die Fliege und die Suppe hervorgegangen. Sowohl der Roman Der Immune wie Die Papiere des Immunen bestehen aus einer grösseren Anzahl kürzerer Texte, welche durch die Figur des Immunen zusammengehalten werden; die einzelnen Texte stellen Varianten menschlichen Lebens dar, MenschenMögliches, wie es einmal heisst.

Im Roman Der Immune wird die Figur des Immunen noch auf einen rudimentären Lebenslauf festgelegt, auch wenn aus diesem keine kohärente Figur entsteht. Der Immune wird uns in verschiedenen Rollen vorgeführt, angefangen beim Säugling über das Stadtkind, das zum ersten Mal aufs Land kommt, er wird uns als bezahlter Liebhaber einer reichen Frau, als Journalist, als Reisender vorgeführt. Er kann als eine Art Mann ohne Eigenschaften gesehen werden, der verschiedene Möglichkeiten ausprobiert. In den Papieren des Immunen werden diese Möglichkeiten um solche erweitert, die die Grenzen der Wahrscheinlichkeit überschreiten: so kann der Immune ins 17. Jahrhundert reisen, im 18. Jahrhundert an einem Leichenmahl eines Marquis teilnehmen, und er kann im Milieu der Schaufensterpuppen verkehren. Wenn der Immune auf diese Weise in den verschiedensten Milieus und Ländern auftreten kann, so hat er alle Eigenschaften eines dynamischen Helden, so wie ihn Lotman definiert, doch erwirbt dieser Held im Gegensatz zum Helden des klassichen Bildungsromans nicht neue Werte, wenn er Grenzen überschreitet, denn die verschiedenen Möglichkeiten des Lebens werden gleichwertig nebeneinandergestellt.

11 Wenn es Grenzen gibt, so sind sie allein auf die Beschränktheit der Menschen zurücführen: Dies wird im Text «In und out» im Immunen dargestellt. Es geht da um Moden im Kulturbetrieb, entweder man kennt die neuste Mode und ist dann «in» oder eben nicht. Als der Immune auf einer Party fragt, was das eben für eine Platte gewesen sei, beweist er mit der Frage, dass er «total out» ist. Der Immune rächt sich, indem er fragt: «Kennt ihr den bolivianischen Minenchor? [...] Das ist im Augenblick der grösste 
Hit in Südamerika». Die andern sind verblüfft: «Nun war der Immune "in", ganz allein, und alle anderen waren "out"; er war "in" dank einer Anstrengung seiner Phantasie» ${ }^{7}$. Die Phantasie ist es denn auch, die immer wieder zur Auflösung der Grenzen führt, nicht nur, wenn der Immune ins 17. Jahrhundert zurückreist, sondern auch wenn er lernt, dass es in Brasilien Spitäler ohne Eisschrank gibt, was er sich mit seiner europäischen Phantasie nicht vorstellen konnte ${ }^{8}$. Wenn der Immune dazu lernt, so sind es nicht andere Werte, wohl aber andere menschliche Möglichkeiten, die er ohne Wertung wahrnimmt. Er erwirbt sich im Laufe seines Lebens die Haltung ei nes Ethnologen, der feststellt: «da machen sie es so, und anderswo anders»?

Sich in diesem Sinn als Ethnologe zu betätigen, bedeutet die völlige Offenheit der Figur, die sich weder durch Herkunft noch Ausbildung in ihren Möglichkeiten beschränken lassen will. Darum legt sich z.B. der Immune eine Unzahl von Vorfahren zu. Statt eines «Lob des Herkommens» schafft er sich eine «Inflation der Vorfahren», indem er ausrechnet, wie viele Vorfahren er seit der Gründung der Eidgenossenschaft habt, diese werden noch erweitert durch diejenigen aus Papier: «Je mehr Vorfahren er sich zulegte, umso mehr befreite er sich von ihnen $»^{10}$. Dass die Inflation der Vorfahren mit der Auflösung der Grenzen zusammenhängt, zeigt sich in der Papieren des Immunen, wo es heisst: «Wir hatten kein Talent für Grenzen entwickelt; das hing wohl damit zusammen, dass wir ohne Wurzeln, aber mit Füssen leben wollten» ${ }^{11}$. "Mit Füssen leben" heisst, in der Welt herumziehen und sich alle Möglichkeiten offenhalten, heisst zugleich sich auf keine festlegen, keine Grenzen ziehen, in diesem Sinn gleicht der Immune dem Mann ohne Eigenschaften, beide sind sie auch Intellektuelle ${ }^{12}$. Wie der Mann ohne Eigenschaften prüft er für sich die verschiedensten Rollen, um sie alle abzulehnen, denn eine Rolle annehmen, Wesse sich beschränken. Nur nebenbei sei darauf hingewiesen, dass Ulrich auch ausdrücklich nicht auf seine Vorfahren festgelegt wird, er trägt keinen Familiennamen und die ihm vom Vater zugedachte Rolle lehnt er ausdrücklich ab. Die Offenheit des Immunen wird als die Eigenheit dargestellt, nirgends ganz dazuzugehören: «Als Intellektueller gehörte er zu einer Minorität des Volkes, redete aber immer wieder vom Volksganzen: Unter Umständen waren ihm Intellektuelle anderer Länder näher als der Mann, der ihm die Post brachte. [...] Er gehörte den verschiedensten Majoritäten und Minoritäten an, gleichzeitig und nebeneinander, er hatte die gleiche Zugehörigkeit als Frage der Majorität und als solche der Minorität erlebt, und diese Zugehörigkeit konnte sich je nach Situation verschieben, und sich im Laufe der Zeit ändern»" ${ }^{13}$. Ein Held, der auf diese Weise je nach Blickwinkel $\mathrm{zu}$ immer wechselnden Gruppen gehört, besitzt keinen festen und begrenzten Staz von Eigenschaften mehr, die gewisse Handlungen ausschlössen, andere aus seinem Charakter folgen Hessen. Man erinnert sich daran, dass Musil jedem Menschen neun Charaktere zuschrieb, was so viel heisst, wie ihm keinen zuzuschreiben, denndiese neun «lösen ihn auf $»^{14}$.

Nachdem wir uns mit dem offenen Helden befasst haben, stellt sich die Frage, in welcher Weise die Grenzenlosigkeit der Welt dargestellt wird. In den Papieren des Immunen wird die Relativität aller Standpunkte durch den Umgang des Immunen mit dem Globus veranschaulicht. Der Immune liebt es, Geschäfte zu besuchen, wo Globen verkauft werden: «er versetzte dem Globus einen Stoss, so dass die Erdkugel zu rotieren begann und zwar so rasch, dass die Farben der einzelnen Länder und die der Kontinente und Ozeane in einem bunten Wirbel ineinander übergingen. Sein 
Bravourakt liess die Nabel der Welt und ihre Herzen verschwinden und zauberte alle Reiche der Mitte fort $»^{15}$.

Die Relativität des europäischen Standpunktes wird in mehreren Texten thematisiert, so in jenem, der mit «Die andere Antike» überschrieben ist und der darstellt, wie ein Europäer entdeckt, dass es nicht nur die europäische Antike gibt, sondern dass es auch eine südamerikanische und eine asiatische Antike gibt.

In der Welt des Immunen spielen die Städte eine besondere Rolle. Sie sind ein Ort der unbegrenzten Möglichkeiten, ein Ort einer Freiheit, die weder «Wall noch Graben» garantieren ${ }^{16}$, ein Ort, wo die verschiedensten Menschen zusammenleben:

Wir standen auf einem der Hauptplätze und taten nichts als schauen, wer uns entgegenkam und an uns vorbeiging: Südländer und nordische Typen, Indios und Levantiner, Mulattinen und Mestizen, Mestizinnen und Mulatten, Juden und Farbige jeder Tönung, Germanen, Slaven, Japaner und all die, über deren Herkunft und Zugehörigkeit wir nur Vermutungen anstellen konnten. Was es da alles an Haaren zu sehen gab, an seidenfädigem, krausem, struppigem, blondem, braunem und rotem, wie verschieden die Backenknochen ausgeprägt waren und wieviele Formen die Augen hatten und erst die Vielfalt der Nasen ${ }^{17}$.

Das Zitat ist zugleich ein Beispiel dafür, wie Loetscher sprachlich der Vielfalt der Welt beizukommen versucht. Loetscher liebt Listen und die Aufzählung von Paradigmen. Die RegisterArie der Städte, aus der das Zitat stammt, ist ein besonders deutli dies Beispiel für dieses Verfahren. Als Prinzip der Aufzählung dienen die verschiedensten Gesichtspunkte: die Städte, die nur Namen aus Lautsprechern sind, Städte, die nicht am Weg liegen, «neben den verpassten Städten die aufgeschobenen, aufgespart für das nächste Mal», «Städte, die nötig waren, um in anderen anzukommen» usw. Die Liste par excellence ist das Wörterbuch, das der Immune überaus liebt, weil es ihm erlaubt, all das $\mathrm{zu}$ beschwören, «was es gab und was es hätte geben können ${ }^{18}$. Das Wörterbuch ist zugleich ein Inventar, das auf jede Wertung und Hierarchisierung verzichtet.

17 Noch wichtiger für das Bestreben des Immunen «alles mit allem in Beziehung (zu) setzen ${ }^{19}$, kugelförmig statt flächig zu reden, ist die metaphorische Sprechweise, in dem weiten Sinn, in dem sie Roman Jakobson verstanden hat. Loetscher hat eine ausgesprochene Vorliebe für alle Arten von Ähnlichkeitsbeziehungen: für Vergleiche, Metaphern, Antithesen und Assoziationen. In einem «Der Aufbruch» betitelten Text in den Papieren vergleichen ein Schweizer und ein Portugiese ihre SehnsuchtsKrankheit:

Die eine stammte aus den Bergen und die andere von den Ufern. Der eine kannte das Firnelicht, die Schneeschmelze und die Lawine, und der andere das Flussfeuer, den Schiffsuntergang und die Springflut. Der eine unterhielt einen Bannwald und der andere baute Dämme. [...] Der eine schrie sein Leiden den Bergen entgegen. [...] Und der andere rief sein Leiden ins Meer hinaus, der Wind trug es fort [...]. Als die beiden ihre Sehnsucht verglichen hatten, klopfte Mestre dem jungen Schweizer auf die Schulter und ernannte ihn zu einem Süsswasser-Portugiesen ${ }^{20}$.

Durch diese Metapher verwandelt sich der fremde Schweizer in einen, der dem Portugiesen nicht mehr fremd ist. Loetscher thematisiert auf diese Weise immer wieder das Ähnliche im Fremden, wodurch zugleich das Eigene und das Fremde relativiert werden. Auf ähnliche Weise wird das Dorf der Väter mit dem Dorf des Immunen in Beziehung gesetzt, welches ein Stadtteil von Zürich ist: «Das Niederdorf, das war das Dorf des Immunen ${ }^{21}$. Hier kennen sich die Bewohner wie im Dorf auf dem Land, hier spricht man eine eigene Sprache, in der auch dieses Kapitel des Immunen geschrieben ist. 
Die Metapher als Mittel zur Auflösung von Grenzen wird auch von Proust thematisiert und zwar besonders in den Bildern von Elstir.

Une de ses métaphores les plus fréquentes dans les marines qu'il avait près de lui en ce moment était justement celle qui, comparant la terre à la mer, supprimait entre elles toute démarcation ${ }^{22}$.

Elstir spreche in Ausdrücken des Meers von der Stadt und in Ausdrücken der Stadt vom Meer, heisst es.

Zum Schluss dieser Untersuchung über die Auflösung der Grenzen in Loetschers Werk muss auch noch die Vielsprachigkeit in der Einsprachigkeit genannt werden. Loetscher macht einen ausgeprägten Gebrauch der verschiedensten Textsorten: der Slang des Zürcher Niederdorfs steht neben sachlichen Texten, der Spiegeljournalistenton neben dem Märchen und dem indianischen Erlebnisbericht von der Entdeckung der Schweiz, die gebrauchsanweisung und die Ratschläge des Briefkastenonkels neben den Phrasen, die auf einer Party gewechselt werden. Es gibt hier keine übergeordnete Instanz mehr, welche diese Erscheinungen in eine Hierarchie brächte, auch sie sind eben einfach Manifestationen des grenzenlosen Menschen-Möglichen.

\section{NOTES}

1. - Ju.M. Lotman, Die Struktur literarischer Texte, München 1972, S.311ff (= UTB 103).

2. - A. Döblin: «Mein Buch Berlin Alexanderplatz». Abgedruckt in: Materialien zu Alfred Döblins Berlin Alexanderplatz. Hrsg. von Prangel, Frankfurt 1975, S.43 (= st 268).

3. - Robert Musil: Gesammelte Werke. Hrsg. von A. Frisé, Bd. 2, Reinbek b. Hamburg 1978, S. 140. Hervorhebung R.Z.

4. - Robert Musil: Der Mann ohne Eigenschaften, in Gesammelte Werke, Bd. 1,S.762.

5. - Marcel Proust, A la recherche du temps perdu, Paris, Gallimard, 1965, Bd. 3, S. 693 (Bibliothèque de la Pléiade).

6. - Die Papiere des Immunen, Zürich, 1986, S. 347 ff.

7. - Der Immune, Zürich, 1985, S. 388.

8. - Der Immune, S. 398.

9. - Der Immune, S. 415.

10. - Der Immune, S. 91.

11. - Die Papiere des Immunen, S. 258.

12. - Vgl. Die Papiere des Immunen, S. 401.

13. - Der Immune, S. $365 f$.

14. - Musil : Der Mann ohne Eigenschaften (zit. Anm. 4), S. 34.

15. - Die Papiere des Immunen, S. 257.

16. - Die Papiere des Immunen, S. 188.

17. - Die Papiere des Immunen, S. 189.

18. - Die Papiere des Immunen, S. 190.

19. - Die Papiere des Immunen, S. 258.

20. - Die Papiere des Immunen, S. 216.

21. - Der Immune, S. 68. 
22. - Proust: A la recherche du temps perdu, Bd.l, S. $835 \mathrm{f}$. 